\title{
IDEF method-based simulation model design and development
}

\author{
Ki-Young Jeong ${ }^{1}$, Lei $\mathrm{Wu}^{2}$, Jae-Dong Hong ${ }^{3}$ \\ ${ }^{1}$ Engineering Management Program at University of Houston-Clear Lake (US A); ${ }^{2}$ Software Engineering \\ at University of Houston-Clear Lake (USA); ${ }^{3}$ Industrial Engineering Tech at South Carolina State \\ University (USA) \\ jeongk@ubcl.edu;.wuL@ubcl.edu;jdhong@ms.com
}

Received May 2009

Accepted August 2009

\begin{abstract}
The purpose of this study is to provide an IDEF method-based integrated framework for a business process simulation model to reduce the model development time by increasing the communication and knowledge reusability during a simulation project. In this framework, simulation requirements are collected by a function modeling method (IDEF0) and a process modeling method (IDEF3). Based on these requirements, a common data model is constructed using the IDEF1X method. From this reusable data model, multiple simulation models are automatically generated using a database-driven simulation model development approach. The framework is claimed to help both requirement collection and experimentation phases during a simulation project by improving system knowledge, model reusability, and maintainability through the systematic use of three descriptive IDEF methods and the features of the relational database technologies. A complex semiconductor fabrication case study was used as a testbed to evaluate and illustrate the concepts and the framework. Two different simulation software products were used to develop and control the semiconductor model from the same knowledge base. The case study empirically showed that this framework could help improve the simulation project processes by using IDEF-based descriptive models and the relational database technology. Authors also concluded that this framework could be easily applied to other analytical model generation by separating the logic from the data
\end{abstract}


Keywords: IDEF0, IDEF3, IDEF1X, discrete event simulation, business process

\section{Introduction}

Simulation is one of the most widely used decision aid tools due to its power, flexibility, and robustness. Particularly the discrete event simulation (DES) can model and analyze the behavior of many real life processes such as business processes, supply chain, and manufacturing processes. However, as Ryan et al. pointed out (2006), the simulation modeling often becomes a heavy programming task with the essence of the system being modeled lost in the detailed programming codes. In this way, the essence of the system is visible only to the code developers. This could create several potential problems for those who are involved in a simulation project. For example, it may create a serious information reusability problem. A simulation model is an abstracted representation of a real system to solve specific problems. Hence the information collected and extracted from the real system should be systematically represented and stored for future reuse in the form of systematic descriptions and formats. It may also cause a communication problem between developers and users. Typically users are domain experts who want to experiment with the simulation model to solve domain specific problems. This task requires frequent parameter changes and modification of the model. However, the heavy codes add difficulty to the proper management of this task. If we consider a simulation model development as a project, and if we have a structured systematic tool to support the simulation project, we believe that these problems could be managed. Sheppard (1983) proposed a widely cited "40-40-20" simulation model development time rule which states that analyst's time should be distributed as follows for a successful simulation project: (1) $40 \%$ to requirement collection phase such as problem formulation, project planning, conceptual model development, and data collection; (2) $20 \%$ to model translation phase; (3) $40 \%$ to experimentation phase such as model verification, validation, implementation, and interpretation. Hence, for successful implementation of any simulation project, it is particularly important to have a right approach to the requirement collection and the experimentation phases. Hence, this paper intends to provide an integrated framework for those two phases in a simulation project. 
The process description methods could play an important role in the simulation requirement collection phase. Although many process design, analysis and modeling (DAM) methods have been developed, using these methods in isolation non-methodological approach - often fails to capture critical system behaviors due to the complexities and component interactions within the system. A methodological approach - systematic usage of a suite of methods - has a greater chance of success at representing critical system behaviors since it can account for diverse aspects of DAM activities such as information, function, and process interactions by a systematic and integrated usage of methods. IDEF (Integrated DEFinition) is a suite of descriptive modeling methods within which several different modeling languages are defined to describe systems from different perspectives. First, since IDEF is a well defined suite, it is considered to be easier to implement a methodological approach with the IDEF suite rather than with a completely different set of methods. Second since it is a descriptive modeling method, it could easily abstract and capture the essence of the system. In a typical simulation project, a project team consists of many team members such as system analysts, developers and domain experts. The system analysts collect and refine requirements with assistance from domain experts. This is an iterative communication process among all members. The 'descriptiveness' of IDEF methods could make this communication process easier and smoother than any other nondescriptive methods. For these reasons, IDEF methods have been a continued research subject.

The first category of the IDEF method related research attempted to build a generic and conceptual descriptive model using IDEF suites in a specific domain (Ang et al., 1994; Zhang et al., 1996). Another category proposed a way to generate an analytical model from a specific IDEF model. For example, an IDEF3 method has been used to generate simulation models using Witness simulation software (KBSI, 1995) and using Arena software (Resenburg et al., 1995). Jeong et al. (2008) developed a scheme to integrate the IDEF3 with a general open queuing network where IDEF3 works as a knowledge repository. The third category employed multiple IDEF methods and attempts to reuse common system knowledge among the different IDEF methods. For example, Lingzhi et al. (1996) proposed a scheme to integrate IDEF1 with IDEF0 for a computer integrated manufacturing information system design. Chen et al. (2004) also proposed a scheme to develop the enhanced IDEF1 information model based on the IDEF0-based process information, 
which could serve as a base representation for an information model. This paper covers both the second and the third category together. It is an extension of Cho et al. (1999), KBSI (1995), and Chen et al. (2004) in that it attempts to provide an integrated framework of IDEF method-based simulation model design and development to help a successful simulation project.

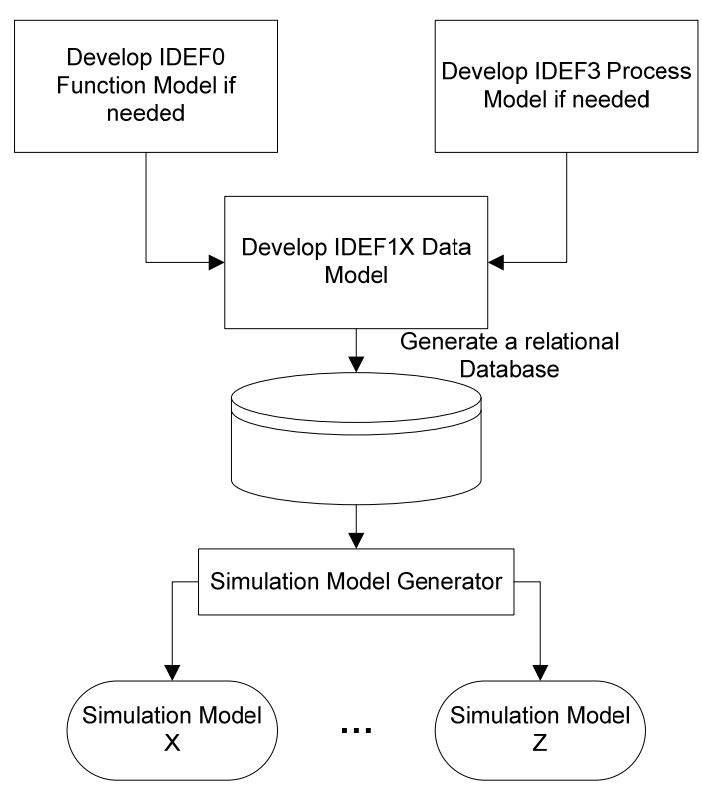

Figure 1. "Conceptual framework of IDEF-based integrated approach"

The experimentation phase is an iterative process to perform model verification, validation, actual implementation, and interpretation that requires another $40 \%$ of analyst's time. The verification, validation, and implementation processes frequently require significant code changes and model modification. It may also require multiple models with different parameter values and diverse scenarios. Hence, it would be helpful to automatically develop diverse versions of a simulation model from a common knowledge base developed through DAM activities. Although it is very difficult to develop a generic database-driven simulation model working for any domain, the domain specific database-driven simulation model development is feasible and useful. In fact, Pidd (1992) pointed out this fact by saying "generic simulators will not wholly replace simulation program for specific application." In this paper, authors suggest a framework where a simulation model is automatically generated from the database obtained as a result of IDEF-based model design and development. Specifically, the functional system knowledge captured by IDEFO and the process system knowledge captured by IDEF3 is used to develop and refine the data model knowledge captured by IDEF1X. Based on 
IDEFIX, a simulation model is generated with a database-driven simulation model generation approach (DBSMGA). In this framework, the IDEFIX model works as a software independent knowledge base where different simulation software could access to generate models using their libraries. The framework is explained in Figure 1.

This framework is claimed to have the following advantages over nonmethodological approaches:

- It facilitates knowledge reusability among different modeling methods during requirement collection phase.

- It can capture diverse aspects of real systems from different perspectives, which improve the accuracy of representation.

- It improves simulation model maintainability since the simulation logic can be changed inside the database as opposed to inside the simulation model.

However, it should be noted that authors do not find it necessary to use this framework for all situations. Instead, this paper claims that this framework has a better chance to lead a successful simulation project by improving communication and knowledge and model reusability. For example, simulation models may be directly built using an icon-based drag-and-drop approach if analysts have sufficient simulation software knowledge. However, based on authors' experience, this direct model building practice without any methodological approach often generates incorrect models due to the lack of communication, improper model abstraction, and inappropriate model management techniques. Particularly, in a large-scale simulation project, it tends to add more difficulty to the proper simulation project management.

\section{I DEF Methods for Business Process Knowledge Capture}

According to Lin et al. (2002), a business process has the following elements:

- A business process has its customers.

- A business process is composed of activities whose objectives are to create values for customers. 
- Activities are performed by actors who may be humans or machines

- A business process often involves organizational units which are responsible for the whole process.

We believe that IDEF methods well support those elements. For example, IDEF0 was designed to capture the 'decisions' and 'activities' of a system (Mayer et al., 1995). Those decisions and activities include information on what functions the system performs, what constraints the functions have, what is needed for functions, and what input and output are meaningful in performing those functions. An IDEFO model is represented with rectangles with four different types of arrows surrounding the rectangles. A rectangle represents a function or activity described in a verbal phrase, and arrows represent (1) "Input" (on the left); (2) "Output" (on the right); (3) "Control" (on the top); and (4) "Mechanism" (on the bottom) called (ICOM) described in a noun phrase to explain the behavior of the function - see Figure 2 below. It also supports the hierarchical decomposition of activities for an appropriate abstraction of a system. We notice that the first three business elements could be supported by IDEFO. For example, IDEFO model could be developed from a specific customer's perspective and context - first element. The business activities are part of system activities - second element. The mechanism in ICOM includes actors - third element.

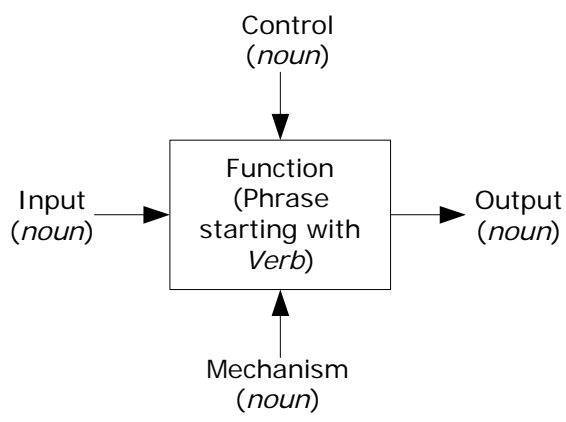

Figure 2. "IDEFO function modeling notation".

IDEF3 is a process capture and description method within the context of a specific scenario (Mayer et al., 1995). The process schematic of IDEF3 has been widely accepted as a medium for process description in industry (Mo et al., 1998). As seen in Figure 3, the process schematic consists of the three main components: (1) "Unit Of Behavior" (UOB); (2) "Junction"; (3) "Link". 


\begin{tabular}{|c|c|c|}
\hline Name & Description & Symbol \\
\hline $\begin{array}{l}\text { Unit of } \\
\text { Behavior } \\
\text { (UOB) }\end{array}$ & $\begin{array}{l}\text { Capture information on what is going on in the system, which } \\
\text { represents a process or an activity }\end{array}$ & \begin{tabular}{|l|l|}
\multicolumn{2}{|c|}{ name } \\
$1 D$ \\
$D$
\end{tabular} \\
\hline Link & $\begin{array}{l}\text { Represent temporal, logical, causal, natural or relational constructs } \\
\text { between UOBs }\end{array}$ & $\rightarrow$ \\
\hline Junctions & $\begin{array}{l}\text { Specify a logical branching of UOBs: } \\
\text { Fan-Out XOR/Fan-In XOR } \\
\text { Fan-Out AND/Fan-In AND } \\
\text { Fan-Out OR/Fan-In-OR }\end{array}$ & \begin{tabular}{|l|l||}
$x$ & $x$ \\
& \\
& $\&$ \\
0 & 0 \\
\end{tabular} \\
\hline
\end{tabular}

Figure 3. "IDEF3 process schematics".

A UOB captures information on what is going on in a system to represent a process or an activity. It is depicted by a rectangle with a unique label. Junctions provide a mechanism specifying a logical branching of UOBs and introduce the timing temporal - and sequencing of multiple processes. Junction types include an exclusive OR denoted by " $X$ ", a conjunctive AND junction denoted by " $\&$ ", and an inclusive OR denoted by "O". Since IDEF3 uses a scenario as a basic organization structure to describe how things work, it could easily fit with the first three elements of a business process. It also supports the top-down and bottom-up modeling sequences and hierarchical decomposition for multiple levels of abstraction. IDEFIX produces a data model that represents the structure and semantics of information within an enterprise or a system, known as business rules. An IDEFIX diagram is refined into three levels of detail: (1) an entityrelationship level; (2) a key-based level; (3) an attribute level. Diverse business rules are specified according to different levels of detail, and the model development is defined by these three level modeling procedures. Figure 4 shows summary of these three levels with symbols. Interested users are encouraged to read (KBSI, 1994) for detailed grammar and graphical symbols for the IDEF1X method.

Lin et al. (2002) also identified ten essential concepts useful in defining a business process, and we creatively used these concepts to investigate the fitness of the proposed IDEF method-based integrated framework for business process simulation model design and development. The results are summarized in Table 1. This table shows all but one concept are handled and represented by this framework. 


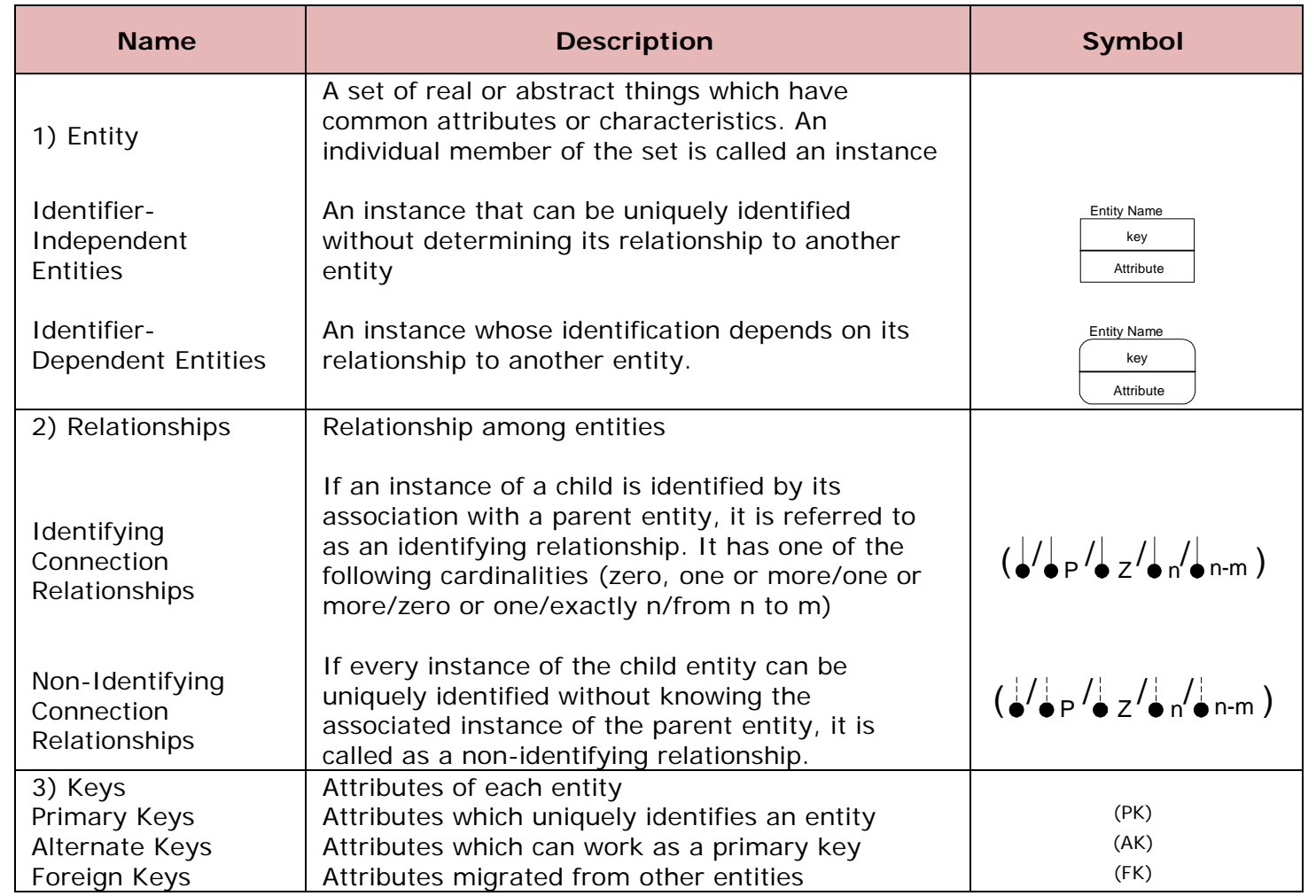

Figure 4. "IDEF1X building blocks and symbols".

\begin{tabular}{|c|c|c|c|c|}
\hline Concepts & Descriptions & I DEFO & I DEF3 & I DEFIX \\
\hline Activity & Task, function or operation & $\checkmark$ & & \\
\hline Behavior & Action, business rules or control & & $\checkmark$ & \\
\hline Resource & Mechanism or location & $\checkmark$ & & \\
\hline Relation & $\begin{array}{l}\text { Relation class, junctions and links, } \\
\text { interaction, and dependencies }\end{array}$ & & $\checkmark$ & $\checkmark$ \\
\hline Agent & Social actor or role & & & \\
\hline Information & Message & $\checkmark$ & & $\checkmark$ \\
\hline Entity & Object represented by attributes & & & $\checkmark$ \\
\hline Event & $\begin{array}{l}\text { Represented by as event objects or } \\
\text { inputs/outputs }\end{array}$ & $\checkmark$ & & \\
\hline $\begin{array}{l}\text { Verification and } \\
\text { validation }\end{array}$ & $\begin{array}{l}\text { Model built as intended? Model well } \\
\text { represents reality? }\end{array}$ & & & \\
\hline $\begin{array}{l}\text { Modeling } \\
\text { procedure }\end{array}$ & Specific procedures to build a model & & & $\checkmark$ \\
\hline
\end{tabular}

Table 1. "Business concepts vs. IDEF methods".

At the same time, it also suggests the intervention from model developers and users are still necessary for verification and validation. In fact, authors do not claim that an automatic model development process could completely replace the insights and knowledge from humans - we even believe that it is not desirable. Instead, this integrated framework should be considered as an aid to support humans and their judgment. 


\section{I DEF1X Data Model Knowledge Base for Simulation Model}

An IDEFIX data model should be robust enough to make the structural and semantic aspects of the simulation included in the model. One way to consider the semantic aspects of the simulation model is to study the simulation ontology, and reflect it within the data model design, since ontology provides the definition of the terminologies and relationship between them. Although several DES software packages are available in the market, they have some common basic structures or objects, while the unique structures are variations of these common structures. If a data model incorporates these structures into its design, it could be shared by different DES software packages.

Table 2 lists some of these basic common structures with their definitions within the context of business process simulation. Note that the neutral terminologies, Generator, Entity, Location, Resource, Queue, and Destroyer are used to avoid favoring a particular simulation language. For example, ED (2001) uses Source, Product, Server or Multiple-Service, Operator, Queue, and Sink while Flexsim (2007) uses Source, Flowltem, Processor or Multiprocessor, Operator, and Sink instead of these neutral terminologies.

\begin{tabular}{|c|l|}
\hline Name & \multicolumn{1}{c|}{ Definition } \\
\hline Generator & A structure that creates entities to populate a model \\
\hline Entity & $\begin{array}{l}\text { A structure that flows through the model to represent customers, orders and any } \\
\text { moving items in the model. }\end{array}$ \\
\hline Location & $\begin{array}{l}\text { A structure that interacts with an entity. This interaction is called a service, and it } \\
\text { usually delays the progress of an entity through the model. }\end{array}$ \\
\hline Resource & $\begin{array}{l}\text { A structure that may be required by an entity or a location to provide a service. } \\
\text { The difference between a location and a resource is that a location does not move, } \\
\text { and a resource is moving toward a location when it is requested. }\end{array}$ \\
\hline Queue & $\begin{array}{l}\text { A structure that stores entities. The queue is awaiting service, not receiving } \\
\text { service. }\end{array}$ \\
\hline Destroyer & A structure that destroys entities \\
\hline
\end{tabular}

Table 2. "Basic simulation objects and definition".

Figure 5 shows one possible mapping between an IDEFIX data model and corresponding neutral simulation structures (objects). The data model has multiple - one or more, denoted by P - Order/Product, Office/Shop, Employee/Equipment or Operator, and Storage/Queue objects. Each of which is mapped to Entity, Location, Resource and Queue object, respectively, in the simulation model. Note that since Generator and Destroyer are purely functional objects for an entity creation and destroy respectively, they do not need to be included in a data model. 
Once a data model is built with consideration of simulation structures, the database can be easily created from which multiple simulation models could be automatically developed.

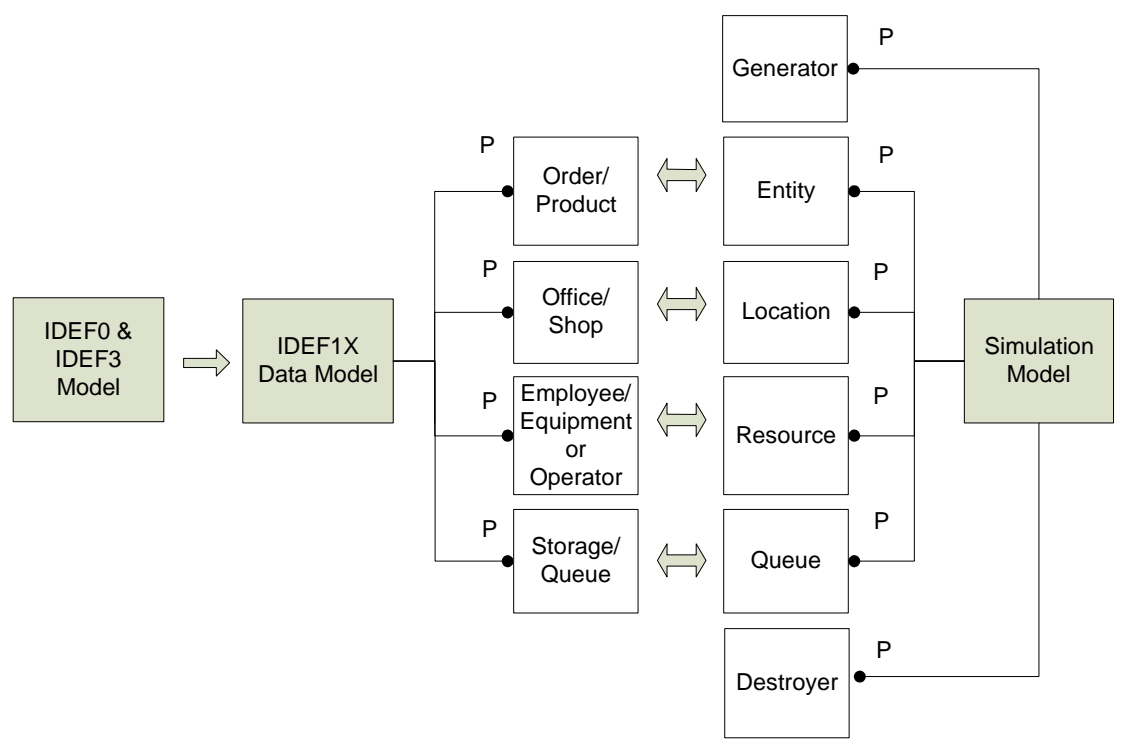

Figure 5. "Simulation and data model mapping".

From a simulation perspective, the data model characterizes the required functionalities of simulation libraries. Hence if those functionalities are not supported by specific target simulation software, those functionalities should be developed to make the database-driven simulation model development easier.

\section{Database-Driven Simulation Model Development}

Most simulation software provides its own script language with structured query language (SQL) and Open Database Connectivity (ODBC) capability. Hence, the interface between the simulation and database management system (DBMS) becomes easier but it still requires some coding efforts. In this study, both Flexsim and ED libraries were adopted to implement DBSMGA since both provide the Object-Oriented customized library development capability in addition to the rich standard libraries. The customized library development capability is supported using Flexscript (Flexsim's script language) or $\mathrm{C} / \mathrm{C}++$ in case of Flexsim and 4D Scripts in case of ED. Each object (library) consists of a set of attributes (characteristics) and methods (functions) that can be implemented when an associated event handler is activated in the library. In case of Flexsim, some typical examples of an event handler are OnReset - triggered when users click the 
reset button, OnMessage - triggered when an object receives a message from other objects, OnEntry - triggered when an entity enters current object, and OnExit - triggered when an entity leaves from a current object. All properties of a class are inherited to the instances of the class when they are created in a model. By combining the standard library and the customized library, users can develop their own user-specific and/or domain-specific simulation software, which makes mapping from the data model to the simulation easier.

In the case study to be discussed later, we first developed a Flexsim model. A customized library named "simulation-generator" was developed to generate simulation models from a database. Note that this corresponds to the Simulation Model Generator in Figure 1. All codes were written in the User event handler and the total lines of the codes are less than 250 including all user-interface and screen embellishment.

\begin{tabular}{|c|c|c|}
\hline NO & Code & Description \\
\hline 1 & $\begin{array}{l}\text { dbopen("MyDSN", "select * from } \\
\text { Equipment",0); }\end{array}$ & $\begin{array}{l}\text { Connected to the database table Equipment through } \\
\text { the DSN defined at MyDSN and perform SQL } \\
\text { statement. }\end{array}$ \\
\hline 2 & $\begin{array}{l}\text { settablenum(“InfoTb", 1, 1, dbgetnum } \\
\text { rows ()); }\end{array}$ & $\begin{array}{l}\text { Store total number of records in database at } \\
\text { cell }(1,1) \text { in InfoTb. "dbgetnumbrows }() \text { " is a key word } \\
\text { to count the number of records in the current } \\
\text { database. }\end{array}$ \\
\hline 3 & $\begin{array}{l}\text { for(int } \mathrm{i}=1 ; \mathrm{i}<=\text { dbgetnumrows(); } \\
\mathrm{i}++)\{\end{array}$ & $\begin{array}{l}\text { Repeat the function defined at }\{\} \text { dbgetnumrows() } \\
\text { times. }\end{array}$ \\
\hline 4 & $\begin{array}{l}\text { createinstance(node("/SHOP- } \\
\text { Equipment",library }()) \text {, model( }) \text { ); }\end{array}$ & $\begin{array}{l}\text { Creates an instance of the SHOP-Equipment class } \\
\text { (library) and places it in the model. Note that SHOP- } \\
\text { Equipment is a customized library. }\end{array}$ \\
\hline 5 & $\begin{array}{l}\text { setname(last (model }()) \text {, dbgettablecel } \\
\mathrm{I}(\mathrm{i}, 1)) \text {; }\end{array}$ & $\begin{array}{l}\text { Change the name of an instance using the name } \\
\text { stored at the Equipment database. } \\
\text { dbgettablecell( }(i, 1) \text { reads the string data stored at } i^{\text {th }} \\
\text { row and } 1^{\text {st }} \text { column }\end{array}$ \\
\hline \multirow[t]{2}{*}{6} & $\begin{array}{l}\text { createinstance(node("/SHOP- } \\
\text { Buffer", library ()), model()); }\end{array}$ & $\begin{array}{l}\text { Creates an instance of the SHOP-Buffer class } \\
\text { (library) and places it in the model. This serves as a } \\
\text { queue for equipment. }\end{array}$ \\
\hline & $\begin{array}{l}\text { setname }(\text { last }(\text { model }()) \text {, concat }(\text { dbget } \\
\left.\left.\text { tablecell }(\mathrm{i}, 1), " \mathrm{Q}^{\prime \prime}\right)\right) ;\end{array}$ & $\begin{array}{l}\text { Define the name of the queue in front of equipment. } \\
\text { Concat connects multiple strings. }\end{array}$ \\
\hline 7 & $\begin{array}{l}\text { contextdragconnection( prev(last(mo } \\
\text { del()), last(model()), "A"); }\end{array}$ & $\begin{array}{l}\text { Connect the output port of queue to the input port } \\
\text { of the equipment }\end{array}$ \\
\hline 8 & Set_Equipment_Attributes, & set equipment attributes (user-defined function) \\
\hline 9 & Set_Queue_Attributes\}; & $\begin{array}{l}\text { set queue attributes (user-defined function) and end } \\
\text { of For statement }\end{array}$ \\
\hline 10 & Dbclose(); & Close equipment database \\
\hline
\end{tabular}

Table 3. "Partial pseudo code in simulation model generator".

Table 3 shows the partial pseudo-codes to create instances of a library object from Equipment table to explain how to read the database through ODBC (lines 1 and 10) and create objects (instances of a library) and connect them using the ports 
(lines 2-9). One record in Equipment table represents one machine instance whose field consists of (equipment ID, equipment Name, Capacity, MTBF, MTTR etc). Lines 8 and 9 are calling user-defined functions to define the attributes of all instances. It is important to recognize that a model is a set of instances of all classes (or objects) located in the library. These classes may be provided by vendors or newly created by users. In this study, the "SHOP-Equipment" object and "SHOP-Buffer" object were created in the library by authors using Flexscript to facilitate the mapping from the data model to Flexsim.

\section{Guidelines for Knowledge Reusability among IDEF methods}

Developing a descriptive model using IDEF methods requires a feedback loop for obtaining consensus and confirmation from domain experts. Considering the fact that each IDEF uses a different modeling language to capture different perspectives of the real systems, the captured knowledge reuse among different IDEF methods has proven difficult to generalize. However, the importance of the knowledge reuse is critical - remember 40-40-20 rule, and how much time is required for requirement collection and experimentation? Based on our experience, the following guidelines seem to be useful in the knowledge reuse among IDEF0, IDEF3 and IDEFIX. As stated previously, IDEFO represents the functional behavior of a system through four different types of data (ICOM) and a set of activities. The ICOM data could be information, objects or anything described in a noun phrase. Hence, some of the IDEFO data may be represented as an object or an attribute in the IDEF1X model. Although IDEFO is not designed to capture the temporal relationship among activities, some functional aspects of the system may include the temporal relationship among activities. Hence, if this happens, some activities in IDEFO could be also represented in the IDEF3 diagram. Guidelines recommended for knowledge reusability among IDEF methods include:

- An IDEFO modeling is recommended first since it provides overall system level knowledge.

- Then, an IDEF3 modeling is recommended if temporal information among activities is needed. Note that some descriptions in UOBs may provide a clue for attributes and objectives in an IDEF1X model. 
- With help of IDEFO and IDEF3, the detailed IDEF1X model could be developed.

- A "Mechanism" in IDEF0 may easily turn out to be an object in IDEF1X since "Mechanism" often includes resources - a typical object in an IDEF1X model.

- The "Constraint" in IDEF0 may provide a logical object in IDEF1X since this "Constraint" often represents the business rules of a system.

- Check if there is any noun in the verbal phrase (function) in an IDEF0 model that needs to be translated into an attribute or object in an IFED1X model. Since a verbal phrase explains the behavior of the function, some nouns used in the phrase may convey meaningful information for a data model.

\section{Case Study}

This paper employs a case study of a semiconductor fabrication process to illustrate the concepts and framework stated in this paper. The IDEF1X data model created for this case study could also apply to many real-life business process problems. This case study originally came from Deuermeyer et al. (1993), and it was adopted here since it involves very complex real-life business processes. This case analyzes 172-step semiconductor wafer fabrication processes with six workareas - CLEAN, STRIP, IMPLANT, DEPOSIT, LITHO and ETCH. These areas perform wafer cleaning, stripping, ion implantation, deposition, lithography and etch operation, respectively. Each work-area consists of machines and operators. An operator is required for wafer-loading and unloading operations at each machine. In addition, when different wafer-lots are loaded, the set up is required. The different operations may have different processing times even though they are performed in the same machine. Each product type can have its own routing as in a general job shop. However, most operation sequences are similar across product types in the wafer fabrication process. For example, all wafers start their operations from CLEAN area and finish at DEPOSIT area after several intermediate operations. One of the typical operation sequences is cleaning, litho, implantation, striping, deposition, etching, striping and deposition again. The important shop information such as the number of machines and operators, MTBF (mean time between failure), and MTTR (mean time to repair) is summarized in Table 4 . Note 
that both MTBF and MTTR are exponentially distributed, denoted by expo(). Two virtual areas were added to indicate the starting (START) and ending (END) of the process. Note that the second column consists of 8-tuples and each of which denotes the number of visits to each work-area. This 8-tuples are arranged in the orders of START, CLEAN, STRIP, IMPLANT, DEPOSIT, LITHO, ETCH and END. It is assumed that this shop is operating for 24 hours with 3 eight-hour shifts due to high capital equipment. The main performance measure for this shop is the system cycle time.

\begin{tabular}{|c|c|c|c|c|c|}
\hline $\begin{array}{c}\text { Work } \\
\text { Area }\end{array}$ & $\begin{array}{c}\text { \# of Visits to Work- } \\
\text { Area }\end{array}$ & $\begin{array}{c}\text { No. of } \\
\text { Machines }\end{array}$ & $\begin{array}{c}\text { No. of } \\
\text { Operators }\end{array}$ & MTBF (hrs) & $\begin{array}{c}\text { MTTR } \\
\text { (hrs) }\end{array}$ \\
\hline START & $(0,1,0,0,0,0,0,0)$ & & & $\operatorname{expo}(42.18)$ & $\operatorname{expo(2.2)}$ \\
\hline CLEAN & $(0,3,0,0,15,1,0,0)$ & 4 & 1 & $\infty$ & 0 \\
\hline STRIP & $(0,2,0,0,1,11,9,0)$ & 3 & 1 & $\operatorname{expo}(55.18)$ & $\operatorname{expo(12.86)}$ \\
\hline IMPLANT & $(0,1,6,1,0,0,0,0)$ & 5 & 1 & $\operatorname{expo}(75.93)$ & $\operatorname{expo}(3.88)$ \\
\hline DEPOSIT & $(0,2,0,0,8,9,8,1)$ & 20 & 3 & $\operatorname{expo}(100)$ & $\operatorname{expo}(2.78)$ \\
\hline LITHO & $(0,5,12,7,2,33,6,0)$ & 33 & 4 & $\operatorname{expo}(62.91)$ & $\operatorname{expo(9.35)}$ \\
\hline ETCH & $(0,5,5,0,3,10,6,0)$ & 28 & 3 & & \\
\hline END & $(0,0,0,0,0,0,0,0)$ & & & & \\
\hline
\end{tabular}

Table 4. "Facility data by work-area".

Based on the description above, we attempt to build an IDEFO model according to the first guideline in the previous section. Each area could be modeled as an activity in IDEF0, and these activities are connected with each other through wafers. For example, Figure 6 shows part of the IDEF0 model in the wafer strip area with its decomposition to show the wafer strip process in detail. For any machine, when a batch of wafers (job) arrives, the operators are responsible for selecting the proper job according to the pre-determined dispatching rule that decides the job processing sequence at the machine. Once a job is selected, it is loaded onto a machine, and the set up occurs if the job's lot number is different from that of the previous job. Once it finishes its operation, the wafers are unloaded and are ready to move to the next destination. The wafer changes its status over time as seen in the figure. For each area, these wafer processes are repeated. 


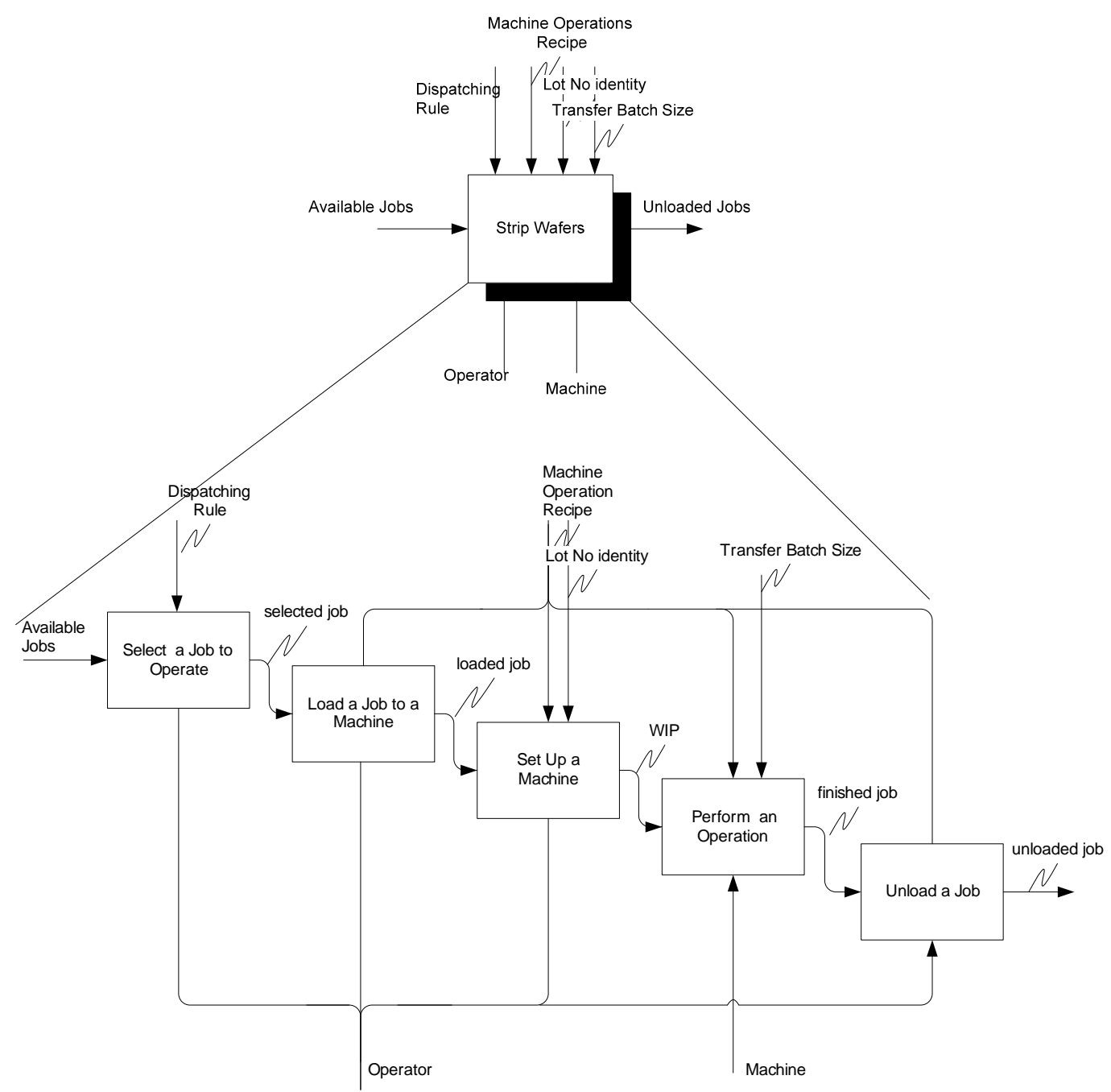

Figure 6. "Function model of a fabrication process in strip area".

Next, according to the second guideline, we tried to consider building an IDEF3 process model. All 172 steps are needed to be represented by IDEF3 model. In this case, the IDEF3 model is same as the process plan of a wafer fabrication, whose routing was already depicted in Deuermeyer et al. (1993). Hence, it was not repeated here. According to the fourth guideline, both an operator and a machine mechanism - are considered as important objects, and they are incorporated into the IDEFIX data model as an object. According to the fifth guideline, the constraint information, Dispatching Rule, is also captured within the data model since it is considered as an important factor affecting the cycle time from the shop scheduling perspective. The same is true for the lot size (Transfer Batch Size) constraint information. It is also observed that the several functions are controlled by a 
constraint called Machine Operation Recipe, which may contain the operation sequence information for each product.

With the help of IDEFO model and the problem descriptions, the IDEFIX model is defined as in Figure 7. Based on above discussions associated with the IDEFO model, the Equipment, Product, Operator and DispatchRule are first defined as an independent object (entity) with keys and attributes. For example, each equipment needs capacity, MTBF, MTTR, setup time and run time, WorkingShift, Overtime, BufferSize and Dispatching_ID information. The BufferSize defines the size of buffer in front of an equipment holding parts awaiting processing, and the Dispatching_ID is the set of rules defining the sequence of jobs in the queue. Typically, it follows FIFO - First-In-First Out. In this case study, the queue object represented in Table 2 is not handled as a separate object since all queues in front of each piece of equipment are considered as infinite.

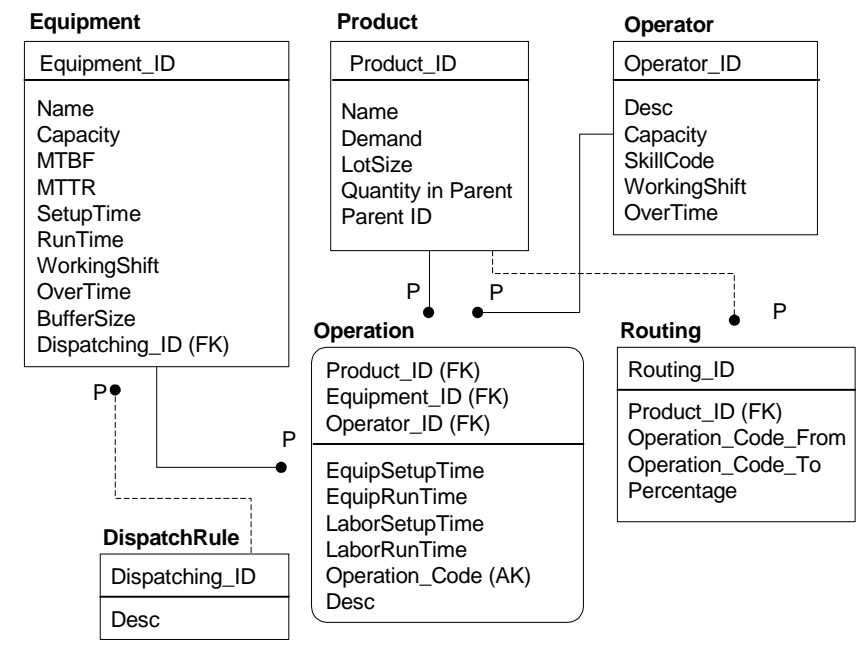

Figure 7. "IDEF1X data model for case study".

The Product object can represent a bill-of-material (BOM) information through Quantity in Parent and Parent ID attributes. The demand and LotSize are attributes that affect the cycle time. The Operation object is defined as a dependent object since it can be uniquely identified only through Equipment, Product and Operator objects. Hence, the relationship between these three objects and an Operation is an identifying connection with one-to-many (one or more) cardinality. However, the relationship between DispatchingRule and Equipment is a non-identifying with one-to-many (at least one) cardinality since DispatchRule_ID is used as a nonprimary foreign key in the Equipment. 
It is important to understand the difference between the Operation and the Routing object. The Operation contains all operation information that describes "who (operator) handles what products with what machines for what time", and the Operation_Code can be used as an alternative primary key. The Routing object is created for simulation model generation to describe the sequence of operations for each product using the information in an Operation object. For each product type (non-primary FK), the source operation code (OperationCode_From) and the destination operation code (OperationCode_To) is described with its corresponding routing probability (Percentage) to support the probabilistic routing view. The Routing object provides the sequence of operations for each product type while these operations are characterized by the Operation object. The prototype data model in Figure 7 was translated into the corresponding MS-ACCESS ${ }^{\mathrm{TM}}$ database using the SmartER ${ }^{T M}$ case tool developed by KBSI (1994). Figure 8 shows a snapshot of the Flexsim simulation model generated from the data model in Figure 7 using the codes in "simulation-generator" library whose partial pseudo-codes are represented in Table 3.

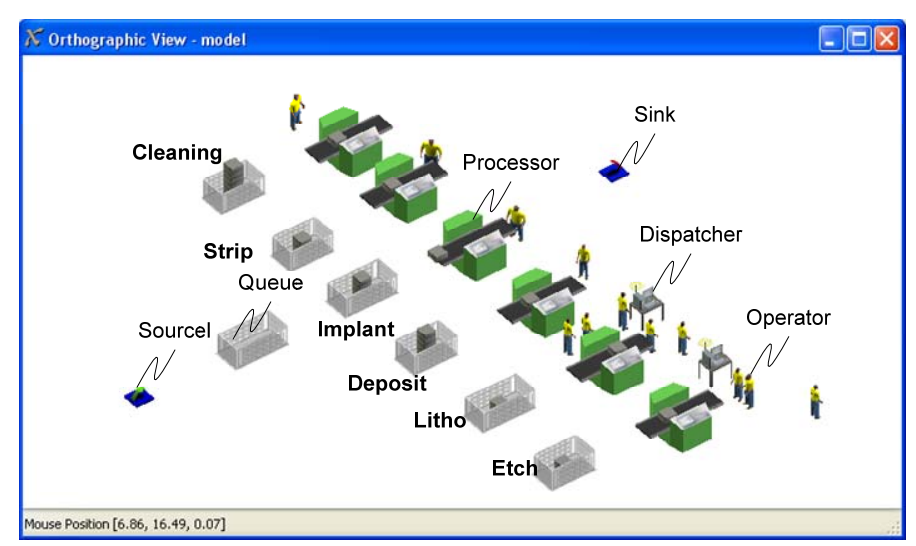

Figure 8. "A snapshot of Flexsim model from database".

In this figure, each of six work-areas is described in bold while the name of an object is represented in a regular letter. When multiple Operators are involved, all operators are directly connected to the Dispatcher object which is directly controlled by a Processor object. Figure 9 shows that of the ED simulation model from the same data model. The library object in ED is called an atom. The queue atom is connected to the operation atom, which is connected to the routing atom that connects the work-areas. The operator control atom (OP CONTROL) is connected to both the operator atom and the operation atom. The first atom 
denoted by "1" represents a product atom corresponding to the Product object in Figure 7 . The queue atom denoted by " 2 " has infinite capacity, which corresponds to a Buffer object. The operation atom denoted by " 3 " contains all equipment information such as MTBF and MTTR as its attributes, and it also includes all suboperations such as loading, set up, cleaning operation and unloading operation.

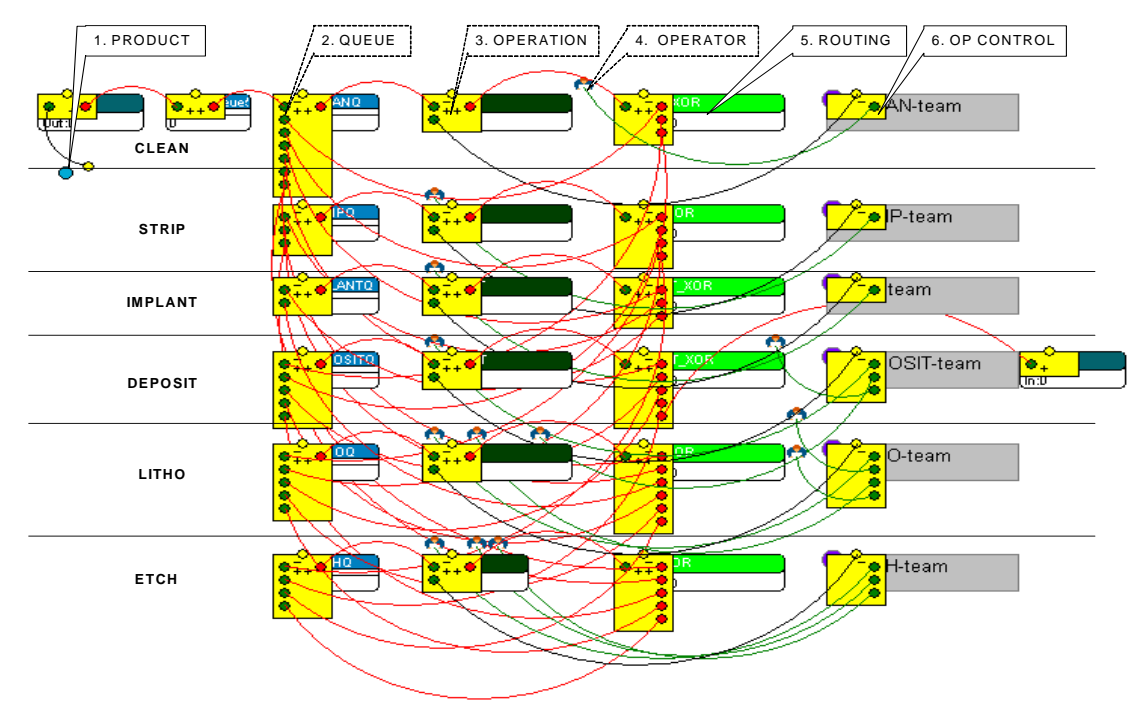

Figure 9. "A Snapshot of ED model from database".

When a sub-operation requires an operator, the atom performing the operation (i.e. cleaning atom) sends an operator-request-message (ORM) to a corresponding operator control atom (OP CONTROL). The operator control atom matches an ORM to an available operator, and it sends available operator(s) to the requesting atom. If there is no available operator for that ORM, it has to wait at the internal message queue inside an operator control atom. Once the (sub) operation finishes, the operator is released from the requesting atom and it becomes available again. All channels are connected using the information in the Routing object, and suboperation information in an operation atom comes from the Operation object in Figure 7. The operator atom corresponds to the Operator object.

This model was executed with the data in Table 4 for 5 times to filter variation, considered as the first alternative (ALT1). Each run has 60,000 simulation hours after 10,000 hours warm up period. Since the sum of the three operators' utilization in CLEAN, STRIP and IMPLANT was around $80 \%$, we created two other alternatives where these three areas have two shared operators (ALT2) and one shared operator (ALT3). The corresponding models were quickly generated again 
from the database by changing the "Operator_ID" in the Operation table without modifying any logic in Flexsim environment for different scenarios. The average cycle time and throughput (number of units produced per day) were compared and displayed in Figure 10 where the bar shows the average cycle time and the line represents the throughput. As seen in the figure, the performance of the second alternative (ALT2) is almost identical to that of the first alternative (ALT1) even with less number of operators, and both outperform the third alternative (ALT3).

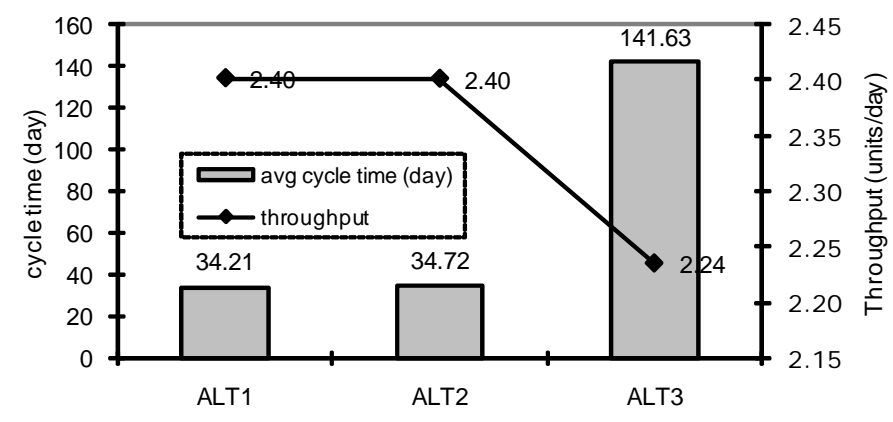

Figure 10. "Alternative comparison".

Through this case study, we showed that the IDEF method-based integrated framework could help improve the process of a simulation project by using IDEFbased descriptive models to capture requirements and to perform the experimentation. The IDEF1X-based data model supported by IDEF0 and IDEF3 could reduce the time and effort for simulation model development and maintenance. Before closing this section, it should be recognized again that the DBSMGA does not depend on any specific simulation software. Any simulation software supporting the ODBC and SQL capabilities could be used. If the software has the capability to customize the standard library, it could also reduce the effort required to map the data model into the simulation model.

\section{Discussion and Conclusion}

In this paper, the integrated framework of IDEF method-based simulation model design and development was provided for a business process. In this framework, the systematic use of IDEFO and IDEF3 for business processes was proposed to help the requirement collection phase in a simulation project. From this systematic use of both descriptive models, the IDEF1X-based data model was created and became a knowledge base from which multiple simulation models could be developed, which could save time and effort in the experimentation phase in a 
simulation project. A case study in a semiconductor manufacturer was conducted to show the feasibility of this framework where both Flexsim model and ED model were generated. This paper also discussed the guidelines to reuse the captured system knowledge among IDEF0, IDEF3 and IDEF1X.

The advantages of this integrated framework are to improve design knowledge reusability among IDEF0, IDEF3 and IDEF1X. It could also significantly reduce simulation model development and maintenance effort. By combining both IDEF methods and the database technologies together, this research significantly improved the previous IDEF based researches in that this framework provided a specific, systematic way to implement and execute the previous IDEF based modeling and design works. Many practitioners and simulation developers have been using the icon-based graphic user interface, and they should be familiar with all icons to develop and use the simulation models - This naturally leads to more focus on the model development phase without the 'descriptiveness' for better communication. However, the framework used here could change this game rule. The use of IDEF methods leads to more focus on the requirement collection phase of the simulation project. Also by using database as a knowledge base, this framework eliminated the dependence on the specific simulation software, and increased the efficiency in the experimentation phase of a simulation project. Authors believe that the results will significantly contribute to the successful use of simulation in the business process area where requirement collection is considered most difficult but important. Another direct advantage of this framework is that this could be applied to any analytical model as long as that model supports the database technology by separating the logic from the data.

The result of this study could provide many new ideas and suggestions to both practitioners and researchers. We summarized these into two categories: IDEF method-based modeling category and the database-based model generation category. Regarding the first category, it would be very useful to automate the knowledge conversion mechanism among IDEF methods to support human judgment and communication during the large scale simulation project. Also although we have provided many rules and insights for this conversion, more research is expected to enrich these lists in a specific domain and/or generic domain. In addition to the IDEF methods, the unified modeling language (UML) could also be considered for this DAM activities since it also supports the diverse modeling approaches from different perspectives. Designing more suitable data 
model and/or database model could be another direct research area in this category since there may be an optimal data model in a specific domain. In the second category, the direct research area includes the development of an integrated simulation model generator through which the same knowledge in the database is transformed into software specific simulation models. In this way, the knowledge reusability will be maximized among simulation software products. Also, integrating all these - both descriptive modeling methods and the database model - within single platform could also be considered as another promising future research area.

\section{References}

Ang, C. L., Luo, M., \& Gay, R. K. L. (1994). Development of a knowledge-based manufacturing modeling system based on IDEFO for the metal-cutting industry. International Journal of Economics, 34(3), 267 - 281.

Chen, P., Caiyun, W., Tiong, R., Seng, K. T., \& Qizhen, Y. (2004). Augmented IDEF1-based process-oriented information modeling. Automation in Construction, 13(6), $735-750$.

Cho, H., \& Lee, I. (1999). Integrated framework of IDEF modeling methods for structured design of shop floor control systems. International J ournal of Computer Integrated Manufacturing, 12(2), 113-128.

Deuermeyer, B. L., Curry, G. L., \& Feldman, R. M. (1993). An automatic modeling approach to the strategic analysis of semiconductor fabrication facilities. Production and Operation Management, 2(3), 195-220.

Enterprise Dynamics. (2001). Reference Guide 4Dscript. Maarssen, The Netherlands.

Flexsim. (2007). Flexsim Simulation Software User Guide Version 4.0, Flexsim Software Products, Inc.

Jeong, K. Y., Cho, H. B., \& Phillips, D. T. (2008). Integration of queuing network and IDEF3 for business process analysis. Business Process Management Journal, 14(4), 471-482. 
KBSI. (1994). SmartER ${ }^{\mathrm{TM}}$ user's manual and reference guide Ver. 2.0, Texas, College Station, Knowledge Based Systems.

KBSI. (1995). ProSim ${ }^{\mathrm{TM}}$ Automatic process modelling for Windows: user's manual and reference guide ver. 2.1. College Station, TX, Knowledge Based Systems.

Lin, F. R., Yang, M. C., \& Pai, Y. H. (2002). A generic structure for business process modeling. Business Process Management J ournal, 8(1), $19-41$.

Lingzhi, L., Leong, A. C., \& Gay, R. K. L. (1996). Integration of information model (IDEF1) with function model (IDEF0) for CIM information system design. Expert Systems With Applications, 10(3/4), 373 - 380.

Mayer, R. J., Benjamin, P. C., Caraway, B. E., \& Painter, M. (1995). A framework and a suite of methods for business process reengineering, in Grove, V. and Kettinger, W. J. (Ed.), Business Process Change: Reengineering Concepts, Methods and Technologies, (pp. 245 - 290). London: I dea Group Publishing.

Mo, J. O. T., \& Menzel, C., P. (1998). An Integrated Process Model Driven Knowledge Based System for Remote Customer Support. Computers in Industry, 37(3), 171-183.

Pidd, M. (1992). Guidelines for the design of data driven generic simulators for specific domains. Simulation, 59(4), 237 - 243.

Resenburg, A. V., \& Zwemstra, N. (1995). Implementing IDEF techniques as simulation modelling specifications. Computers and industrial engineering, 29(1-4), 467-471.

Ryan, J., \& Heavey, C. (2006). Process modeling for simulation. Computers in Industry, 57(5), 437-450.

Sheppard, S. (1983). Applying software engineering to simulation. Simulation Practice and Theory, 10(1), 13-19.

Zhang, J., Chuah, B., Cheung, E., \& Deng, Z. (1996). Information modeling for manufacturing systems: A case study. Robotics \& Computer-Integrated Manufacturing, 12(3), 217-225. 
Article's contents are provided on a Attribution-Non Commercial 3.0 Creative commons license. Readers are allowed to copy, distribute and communicate article's contents, provided the author's and Journal of Industrial Engineering and Management's names are included. It must not be used for commercial purposes. To see the complete license contents, please visit http://creativecommons.org/licenses/by-nc/3.0/. 